\title{
Analysis of the angular sensitivity of an innovative particle velocity sensor
}

\author{
J.W. van Honschoten, D.R. Yntema, R.J. Wiegerink, M. Elwenspoek \\ MESA+ Research Institute, University of Twente, The Netherlands \\ j.w.vanhonschoten@utwente.nl
}

\begin{abstract}
In this paper a novel micromachined acoustic sensor consisting of four heated wires is analyzed theoretically and experimentally. The presence of the chip surface of the probe in the vicinity of the wires influences the local fluid flow, while it also affects the temperature distribution in the probe by altering the direction of heat transport. Both effects result into a specific angular dependence of the sensor sensitivity. To explain this specific directionality of the sensitivity, an analytical model is presented that describes both the air flow around the probe and the temperature profile around the heated wires. Acoustic flow measurements on the probe sensitivity are compared with the theory and with numerical simulations on the device, showing good qualitative and quantitative correspondence.
\end{abstract}

\section{INTRODUCTION}

For a complete characterization of a three-dimensional sound field, both the acoustic particle velocity and the pressure have to be determined. By means of a particle velocity sensor complementary to the conventional pressure microphone, a complete determination of the threedimensional acoustic field, including intensity streamlines and different source contributions becomes possible. To this purpose a micromachined particle velocity sensor, composed of two parallel heated wires, of about $1 \mathrm{~mm}$ length and spaced about $300 \mu \mathrm{m}$ apart, has been developed [1,2,3,4]. When subject to an acoustic flow, forces convection leads to a small temperature difference between the wires, which is a measure of the particle velocity. To exploit fully its abilities

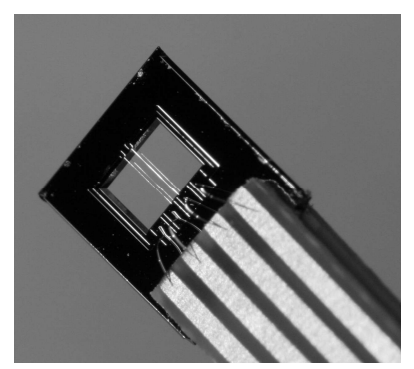

Figure 1. The four wire particle velocity probe, containing four $1 \mathrm{~mm}$ long and $400 \mu \mathrm{m}$ wide sensor wires suspending the rectangular orifice in the chip surface.

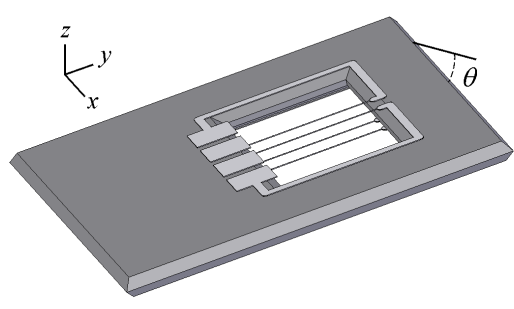

Figure 2. Schematic picture of the probe.

to measure the three-dimensional particle velocity vector, we designed a new device in which four hot-wires in perpendicular directions are integrated in one probe [5] (Fig.1).

For a very accurate determination of the direction of the involved particle velocities, a good understanding of the directional sensitivity of the probe is needed. However, the current designs show a deviation in their directional sensitivity pattern from the ideal 'figure-of eight' response $[2,6]$ that would be the case for free standing wires. Up to now, this deviation has not been fully understood. The purpose of this paper is to analyze this effect and to model the sensitivity for the four wire probe, with the sensor wires placed within a square orifice in the chip surface. In the analysis we take into account all physical aspects that play a role: both the acoustic flow profile around the wires, that is disturbed due to the presence of the printed circuit board acting as a disturbance for the flow, and the local temperature distribution close to the heaters. The chip surface acts as a heat sink that affects the heat transport due to convection and consequently influences the sensor response to particle velocities. We present an analytical model for the directional sensitivity of this novel device, and compare this with experiments and numerical calculations on the flow and temperature distribution in the probe.

\section{EXPERIMENTAL OBSERVATIONS}

The particle velocity sensor of investigation, shown in Fig. 1, consists of four thin wires $(1 \mathrm{~mm}$ long silicon nitride 


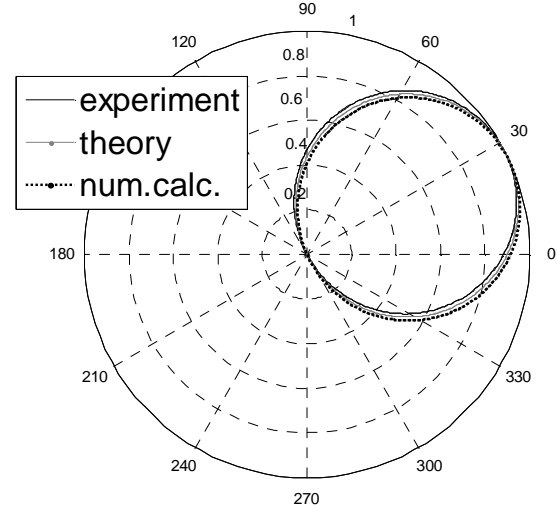

Figure 3. Polar diagram of the sensitivity of the probe at $600 \mathrm{~Hz}$. An 'ideal' figure of eight would have its maximum at $45^{\circ}$. Clearly, the observed polar pattern is rotated with respect to this normal 'figure-ofeight': the measured angular shift $\Delta$ is $18^{\circ}$. Also shown in the figure are the curves according to the theoretic model and the numerical calculations.

beams of $400 \mu \mathrm{m}$ thickness with $100 \mathrm{~nm}$ thick platinum layers on top) suspending across a rectangular orifice of $1 \mathrm{x}$ $2 \mathrm{~mm}$. For the characterization of the probe, it is placed in a $25 \mathrm{~cm}$ long standing wave tube $[2,6]$ with a pressure microphone connected in the tube end, and at the other end a loudspeaker generating a broad band $(0-10 \mathrm{kHz})$ signal. The probe measures the generated standing wave pattern from which its frequency response, i.e. the output signal as a function of the frequency, can be determined. The measured frequency response of the probe is found to be in correspondence with previous measurements on the sensitivity of the particle velocity sensor $[1,9,4]$, showing a low-pass frequency response with a roll-off above $2 \mathrm{kHz}$.

To determine the sensitivity of the probe as a function of the angle of the incoming wave, the device is mounted in an electrostatic step motor, placed in the standing wave tube, and rotated in steps of $3.75^{\circ}$. The measured polar diagram of the angular sensitivity is shown in Fig. 3.

For wires in free space, without any obstructions, the polar representation of the sensitivity resembles a figure-of-eight $[2,6]$. Ideally, the wires of the probe would therefore also show a figure-of-eight pattern, with the long axis of the eight under an angle of $45^{\circ}$ (since the wires are mutually placed under an angle of $45^{\circ}$ with the probe surface, as illustrated by Fig. 1 and 2.) However, it is clearly seen in the polar diagram of Fig. 3, that the figure is rotated with respect to the ideal figure-of-eight; the measured angular shift $\Delta$ is approximately $18^{\circ}$. The measurements show further that this polar pattern is nearly frequency independent in the range from $50-1000 \mathrm{~Hz}$ for all investigated probes.

The reason for the observed angular shift can be explained by different effects. As suggested above, both the influence of the surrounding chip material on the temperature distribution around the wires, and the effect of the chip material on the flow profile in the probe, can play a role. To test this hypothesis and to distinguish between the temperature induced and flow induced effects, different



Figure 4. Angular shift as a function of gap distance $\left(2 l_{x}\right)$ for different types of probes (with balsa wood, and with silicon as surrounding material), and compared with numerical calculations on the devices and with the model. The effects of the temperature, and of the disturbed flow profile, were separately investigated and then added.

series of probes have been designed. In the theoretical section, part III, we can then analyze the data and check the proposed theoretical model.

Two different series of probes have been fabricated, one set with silicon as the surrounding material, and the other with balsa wood. This material has been chosen because of its extremely low heat conductivity compared to that of silicon. Each set of probes comprises of devices with varying gap distances, with the full gap $2 l_{x}$ distance (the width of the orifice) varying between 0.4 and $2.0 \mathrm{~mm}$. All probes are analyzed in the standing wave tube and the shift $\Delta$ of their polar sensitivity is determined. The result is shown in Fig. 4, in which $\Delta$ is plotted versus the gap distance. We see that the balsa based probes show significantly less temperaturerelated effects, resulting in a smaller angular shift. Also depicted in the figure are the curves according to theoretical calculations and to the numerical analysis as described in the next sections.

\section{THEORY}

Ideally, the two parallel heated wires of a particle velocity sensor exhibit a $\cos (\theta)$-dependent response to a fluid flow imposed under an angle $\theta$ with the plane of the wires, since the sensor wires detect the velocity component directed in the plane of the wires only. This $\cos (\theta)$ angular dependence on the incoming flow leads to the above mentioned 'figureof-eight' response. As the measurements show, the polar diagram of the sensitivity of the probe is rotated over a frequency independent angle $\Delta$, with $\Delta$ depending on the geometry of the device and heat conducting properties of the surrounding material of the device. We distinguish the two possible causes for the observed effect: First, the presence of the chip surface in the close proximity of the wires disturbes the air flow, thereby altering the direction of the local particle velocity. Second, the silicon chip acts as a 'heat sink' resulting in a preferred direction of the heat flow generated 


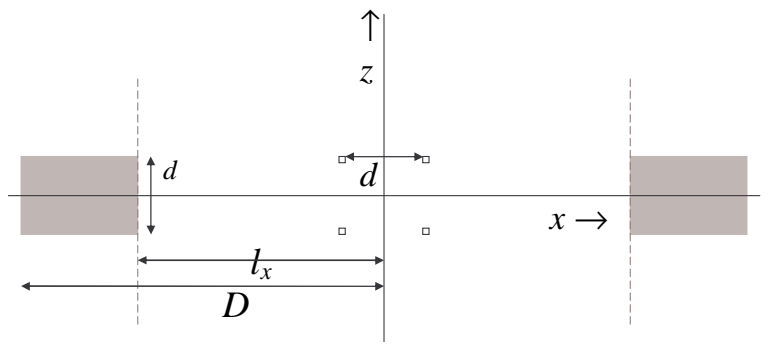

Figure 5. Cross-sectional view of the model geometry for the calculation of the temperature distribution. The thickness of the substrate (the chip) is $d$; its full width is $2 D$, the horizontal distance between the wires is also $d$.

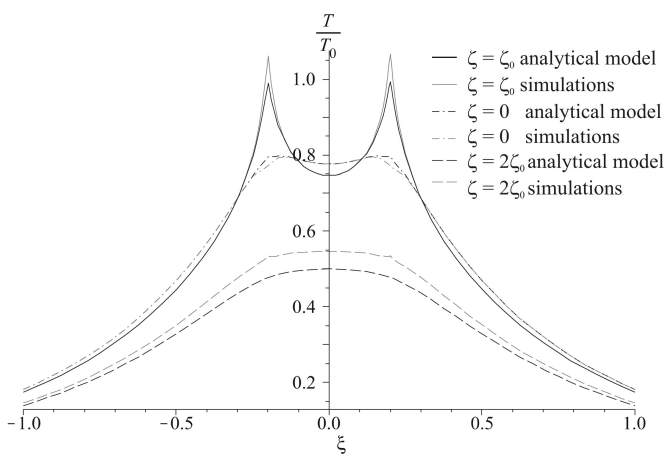

Figure 6. Temperature distribution in the channel along the normalised $x$ axes, according to the calculated expansion for $T$ with $\xi_{0}=0.2, l_{x}=1 \mathrm{~mm}$ and $l_{y}=1.5 \mathrm{~mm}$. For comparison, results from numerical simulations (section IV) are also shown. The temperatures are normalised by $T_{0}$, the temperature at the place of the heaters.

by the sensor wires, to the silicon. Consequently, the temperature distribution around the wires is not completely symmetric anymore as it would be for wires in free space. The purpose of this section is therefore to model both the temperature and the flow profile in the probe.

\section{A. The temperature profile}

To analyze the response of the sensor due to an imposed acoustic flow, the temperature profile around the heated wires has to be calculated. At first, the static temperature distribution in the device is calculated, in the absence of a gas flow. It has been proven [7, 8] that an acoustic wave gives only a small perturbation to this temperature profile, and also that the output signal of the sensor is linearly proportional to the local temperature gradient at the place of the sensor wires. Therefore, the static temperature distribution only, provides sufficient information to deduce the sensitivity properties we are interested in in this paper, in particular the directionality (the polar dependence) of the sensitivity of the device.

In the absence of a moving gas, the temperature distribution around the wires follows from the stationary heat equation

$$
-\vec{\nabla}(k \vec{\nabla} T)=Q
$$

where $k=k(T)$ is the thermal conductivity of air, that is in principle a function of temperature, and $Q$ the heat produced by the heater per unit volume per unit time. We now solve the static temperature $T(x, z)$ for the geometry as shown in Fig. 5. Taking into account the appropriate boundary conditions for $T$ and the heat flux at $x= \pm l_{x}$, we can obtain an expression for the temperature in the device as an expansion in harmonics in normalized variables $\xi=x / l_{x}$, $\zeta=z / l_{x}$. The resulting expansion converges quickly and can be easily calculated numerically. For a specific set of parameters the temperature distribution is shown in Fig. 6, also compared with numerical simulations described in section IV. The thus obtained static temperature profile is the required starting point to find the sensitivity $S$ of the device and its response to an acoustic gas flow. If due to an acoustic wave the gas close to the heaters has a velocity $v$, the temperature around the wires is described by the full heat equation

$$
\rho c_{p}\left(\partial_{t} T+\vec{v} \vec{\nabla} T\right)-\vec{\nabla}(k \vec{\nabla} T)=Q
$$

where $c_{p}$ is the gas heat capacity at constant pressure. The convective term is responsible for the difference in wire temperatures. For small $v$, the solution of this equation is formed by the previously found temperature plus a small correction $\delta T$ proportional to $\vec{v} \cdot \vec{\nabla} \vec{T}$, with $T$ the previously obtained static temperature [7,9]. The sensitivity $S$ is proportional to $\delta T$ and we obtain an expression for the angular dependence $S(\theta)$, with $\theta$ the angle of the incident flow. We find then that the polar representation of $S(\theta)$, the polar figure, is shifted with respect to the 'ideal' figure-ofeight of the sensitivity of free standing wires, by an angle $\Delta$ and that $\Delta$ is fully determined by the ratio of the $x$-and $z$ derivatives of $T$ at the wires (Fig. 3). The calculated shift $\Delta$, due to this temperature effect, as a function of the gap distance is shown in Fig. 4 by the black dotted line.

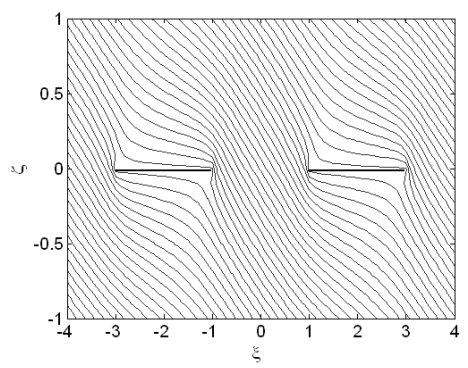

Figure 7. Calculated streamline pattern in the normalized $x$-z-plane (see the cross section of Fig. 5) for an incident flow with an angle of incidence $\theta$ $=\pi / 8$, and a normalized gap distance of the probe equal to 2 .

\section{B. The air flow around the probe}

Due to the presence of the chip surface, the fluid flow itself is influenced as well, and the local angle $\theta$ will in general not be equal to the angle of incidence of the acoustic wave far away from the probe. To analyse therefore the air flow around the probe, one should solve the full Navier 



Figure 8. Numerical simulations on the temperature profile in the probe. Shown are cross sections of the chip, on which thin sheets of material are deposited, displaying the isotherms (normalised by $\mathrm{T}_{0}$ at the wires). Figure left: The material has a heat conductivity $k_{b}=0.04 \mathrm{~W} / \mathrm{m} \mathrm{K}$ (corresponding to the wood that was used in the experiments); right: with $k_{S i}=130 \mathrm{~W} / \mathrm{m} \mathrm{K}$.

Stokes equations for the three-dimensional probe geometry to obtain the complete particle velocity field around the probe. For the relatively complicated geometry of the real probe as depicted in Fig. 1, an exact analytic solution cannot be found. Therefore, we model the sensor chip by a pair of long and thin parallel plates, of which the cross section is shown by Fig. 5. The problem becomes then twodimensional. It has been shown $[8,10]$ that for propagating acoustic waves, the fluid can be considered as incompressible, so that the Navier-Stokes equations yield

$$
\frac{\partial \vec{v}}{\partial t}+(\vec{v} \cdot \vec{\nabla}) \vec{v}=-\frac{1}{\rho} \vec{\nabla} p+v \nabla^{2} \vec{v}
$$

with $p$ the pressure and $v$ the kinematic viscosity. It was shown before $[8,10]$, that for the current values of $v$, the nonlinear term in the equation can be neglected. Using elliptic coordinates [11], the equation can be solved analytically for thin, infinitely long, plates of width $D-l_{x}$ $\left(d \ll\left(D-l_{x}\right)\right)$ For a specific value of $\theta$, the streamline pattern is seen in Fig. 7. From the obtained streamline pattern, we can determine the local velocity of the air at the place of the wires, and determine the angular shift $\Delta$. The values of $\Delta$ due to this flow profile around the probe, are also depicted in Fig. 8 (drawn black line).

\section{NUMERICAL CALCULATIONS}

We used a finite-elements numerical solver, CFD RC $®$, to analyze the temperature distribution and the air flow in and around the three-dimensional probe geometry. Both the Navier-Stokes equations and the heat diffusion equations were solved numerically by constructing a fine mesh of discrete small control volumes in the solution space, defining the appropriate boundary and initial conditions, and solving the equations for each cell. A plane propagating wave of incident angle $\theta$ was defined as entering the outer boundaries, and $\theta$ was varied in the simulations. The dissipated power per wire was set to be $20 \mathrm{~mW}$. Devices with varying gap widths, ranging from $0.2 \mathrm{~mm}<l_{x}<1.8 \mathrm{~mm}$, were investigated. Additionally, to comply with the different devices analyzed in the experiments, the heat conductivity of the chip material was varied in the simulations, ranging from the heat conductivity of air, $k_{\text {air }}=0.0263 \mathrm{~W} / \mathrm{m} \mathrm{K}$, to that of silicon, $k_{S i}=130 \mathrm{~W} / \mathrm{m} \mathrm{K}$, in five linear steps.. The results of the numerical calculations on two suchlike probes are illustrated by Fig. 8 .

A comparative summary of numerical, theoretical and experimental results is presented in Fig. 4. It shows a satisfying correspondence between the theory and the simulations and both are in good agreement with the experimental observations. Besides, it is seen that both the temperature and flow effect play a significant role in the directional sensitivity, whereas the latter effect is dominant.

\section{CONCLUSIONS}

The sensitivity of a novel micromachined acoustic sensor consisting of four heated wires was analyzed theoretically and experimentally. The presented model is seen to be appropriate to describe the angular dependence and the magnitude of the sensor response and in satisfying correspondence with simulations and experiments. Besides, the observed angular dependence of the sensitivity is frequency independent and can therefore easily be corrected for. It is also concluded that the perturbed air flow due to the chip surface is the dominant reason for the observed angular sensitivity. The obtained results allow as well for an optimization of the device.

\section{REFERENCES}

[1] H.E. de Bree, P.J. Leussink, M.T. Korthorst, H.V. Jansen, T.S.J. Lammerink, and M. Elwenspoek, The Microflown: a novel device measuring acoustical flows, Sensors and Actuators A 54, 552-57, 1996

[2] H.E. de Bree, The Microflown Ph-D Thesis, University of Twente, the Netherlands (90-36509262), 1997

[3] H-E. de Bree, T.S.J. Lammerink, M.C. Elwenspoek, J. Fluitman, Use of a fluid flow-measuring device as a microphone, Patent PCT/NL95/00220, 1995

[4] D.R. Yntema, J.W. van Honschoten, H.E. de Bree, R.J. Wiegerink, M. Elwenspoek, A three dimensional Microflown, Proc. of MEMS Conf.(Istanbul) 654-7, 2006

[5] D.R. Yntema, J.W. van Honschoten, R.J. Wiegerink, Integrated 3D Sound Intensity sensor with four-wire particle velocity sensors, Proc. of DTIP, France, 168-170, 2008.

[6] W.F. Druyvesteyn, H-E. de Bree, A new sound intensity probe; comparison to the Bruel \& Kjaer p-p probe, J. audio Eng. Soc., 48, nr $1 / 2,2000$.

[7] V.B. Svetovoy, I.A. Winter, Model of the Microflown microphone, Sensors and Actuators A 86 171-181, 2000

[8] J.W. van Honschoten, D.R. Yntema, V.B. Svetovoy, M. Dijkstra, R.J. Wiegerink, Analysis of the performance of a particle velocity sensor between two cylindrical obstructions, J. of Ac. Soc. of Am., 121, 271122, 2007

[9] J.W. van Honschoten, V.B. Svetovoy, G.J.M. Krijnen, M. Elwenspoek, Analytic model of a two-wire thermal sensor for flow and sound measurements, J. of Micromech. Microeng.,14, 1468-77, 2004

[10] L.D. Landau, Course of Theoretical Physics, 6 Fluid Mechanics, $2^{\text {nd }}$ ed., Butterworth Heinemann, Oxford, 2003

[11] H.Lamb, Hydrodynamics, $6^{\text {th }}$ ed. Cambridge Press, 1975. 